\title{
Image Forgery Detection based on Local Descriptors and Block-Matching using Clustering Technique
}

\author{
Shikha Dubey \\ M.Tech (Information technology) \\ LNCT Bhopal,India
}

\author{
Anshul Sarawagi \\ Prof \& Head (IT) \\ LNCT Bhopal,India
}

\author{
Manish Shrivastava, PhD \\ MANIT Bhopal,India
}

\begin{abstract}
In current decade, digital images are in use in a wide range of applications and for multiple purposes. They also play an important role in the storage and transfer of visual information, especially the secret ones. With this widespread usage of digital images, in addition to the increasing number of tools and software of digital images editing, it has become easy to manipulate and change the actual information of the image. In this detection technique used texture feature of image. For the texture extraction of image used wavelet transform function, these function is most promising texture analysis feature. For the selection of feature generation of pattern used clustering technique. Clustering technique is unsupervised learning technique process by iteration. The proposed methods are evaluated on a number of original and forged images. According to our experimental results the proposed methods are quite attractive. The forgery is done with just copy-move, copy-move with rotation, with scaling, and reflection. In this process, an image database that consists of original and forged images is also developed. The proposed method achieves $100 \%$ accuracy in just copy-move forgery (without any change in the size or characteristics of the object) forgery without post-processing and $98.43 \%, 86.58 \%$, and $95.12 \%$ accuracies in copy-move forgery with rotation, scaling, and reflection, respectively.
\end{abstract}

\section{Keywords}

Image Forgery, Feature Extraction, clustering.

\section{INTRODUCTION}

Image forgery detection is a new area of research in digital image forgery detection technique. In image forgery detection technique various algorithms are used such as statically method, transform based method and feature selection based method. Now a day's used texture feature based image forgery detection[1,2]. The major issue in texture based image forgery detection is measure the correlation of coefficient similarity. But, in today's digital age, it has become easy to change the information represented by an image without any visible traces. But the truth is that with the simplicity of digital image manipulation provided by the development in computer technology, we have to be aware about what we are seeing. Computer hardware and software (such as Adobe Photoshop, GNU Image Manipulation Program "GIMP") today offer the ability of digital image manipulation $[4,5,6]$. The purpose of forgery and manipulation of digital images in many cases is to intentionally affect the awareness of the recipient. In other words, it means that the credibility of digital images is questioned and their content integrity can no longer be fully trusted[7]. We believe that, recovering the community confidence toward digital image contents is very important. Texture based image forgery detection have some limitation related the process of feature selection and region selection of coefficient block[8]. The major problem is measure the similarity of forgery and original image. Optimal feature selection for the purpose of detection Noise value of image equal to higher intensity value of actual image Region of forged image are not precise. The detection of forgery in color image is very difficult due to high intensity of pixel value. Here we used clustering technique by graph prototype collection of data for the process of clustering[9]. For the generation of cluster for the process of detection feature extraction process are required. For the extraction of feature used wavelet transform function. Wavelet transform function well knows feature extraction technique. The wavelet transform gives the texture feature of forged image. After the generation of feature used clustering technique for the generation of cluster for the formation of matching block $[10,11,12]$. Section-II gives the information of feature selection. In section III discuss the proposed method. In section IV discuss the discuss experimental analysis. Finally, in section $\mathrm{V}$ conclusion and future scope.

\section{FEATURE SELECTION}

In the feature selection method the features are either picked manually from the texture feature matrix or by using a specific feature selection tool. The most suitable features are selected by handpicking from the feature spectrum based on the prior knowledge about the environment that the image forgery is used. For example

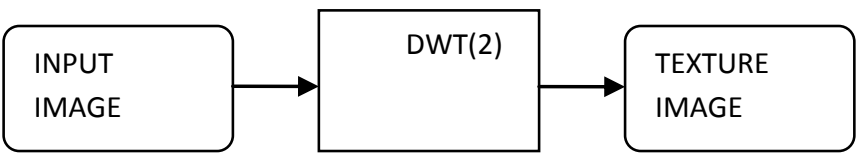

Figure 1: feature selection process in feature variable.

\section{PROPOSED METHOD}

In this section discuss the proposed algorithm for image forgery detection based on clustering technique. In the process of image forgery k-means clustering technique is applied. The $\mathrm{k}$-means clustering technique is very efficient for the creation of block pattern[16]. After the creation of block pattern used block matching process. For the purpose of clustering used texture feature data for forged and original image. Here discuss some algorithm steps for the process of cluster generation and matching process.

1: $\quad$ Forimage $=(\mathrm{X}, \mathrm{C}) \leftarrow$ empty

2: $\quad$ C_list $\leftarrow$ K-means $\left(C i \_l i s t, \mathrm{~K}_{\text {guto }}\right)$

3: Input $C_{-}$list $X$, the clustering number pn , texture scale $\mathrm{XN}$, probability Forimage $\mathrm{P}$ stop conditions $\mathrm{cS}$;

4: Code the data in real number and initialize texture matrix $\mathrm{S}(\mathrm{i}), \mathrm{i}=0$ at random; 
5: Evaluate the all individual in the current instant $\mathrm{D}(\mathrm{s})$;

6: $\mathrm{CR}$ clustering requires number of cluster center, which way thrashing of data of waiting cluster. Hence the fitness function of algorithm is determined by $\mathrm{f}(\mathrm{x})$.

7: $\quad G(s)=\frac{N(s)}{D(s)}=\frac{\sum_{i=0}^{n-1} A_{i} s^{i}}{\sum_{i=0}^{n} a_{i} s^{i}} \quad$ Umpire the termination conditions. If the termination situation are satisfied, then turn to step 9 , if not, turn to step 10 ;

8: Crack to find and compute the number clustering centers.

9: find final block of forged image

10: Take the CR matching on population $\mathrm{P}$ (i) and generate the next generation $A(i+1)$. Then turn to step

11: for $h \in A(i+1) d o$

12: h.nn $\leftarrow \mathrm{CR}(\mathrm{A}(\mathrm{i}+1)-\{\mathrm{h}\})$

13: h.sc $\leftarrow$ Compute-SC (h, h.nn)

14: FORIMAGE $\leftarrow$ FORIMAGE $\boldsymbol{U}_{\{\mathrm{h}\}}$

15: FORIMAGE $\leftarrow$ FORIMAGE $\bigcup_{\{\text {h.nn }\}}$

16: if h.sc $<$ th $_{\mathrm{sc}}$ then

17: $\mathrm{E} \leftarrow \mathrm{E} \cup\{($ h,h.nn $)\}$

18: End if

19: end for

20: count $\leftarrow$ Matrix for each pair of components $(\mathrm{g} 1, \mathrm{~g} 2)$ $\in \mathrm{G}$ do

21: $\mu_{1} \leftarrow$ mean-dist (g1), $\mu_{2} \leftarrow$ mean-dist (g2)

22: if $\frac{\mu_{1}+\mu_{2}}{2 * \text { centroid_dist }(g 1, g 2)}>1$ then $\mathrm{g} 1 \leftarrow$ Merge $(\mathrm{g} 1, \mathrm{~g} 2)$

23: end for

24: N_type $\leftarrow$ empty

25: for $\boldsymbol{x} \in \mathrm{N}$ list do

26: hॅPseudopointOf $(\boldsymbol{x})$

27: estimate detection rate

28: FRR

29: end for

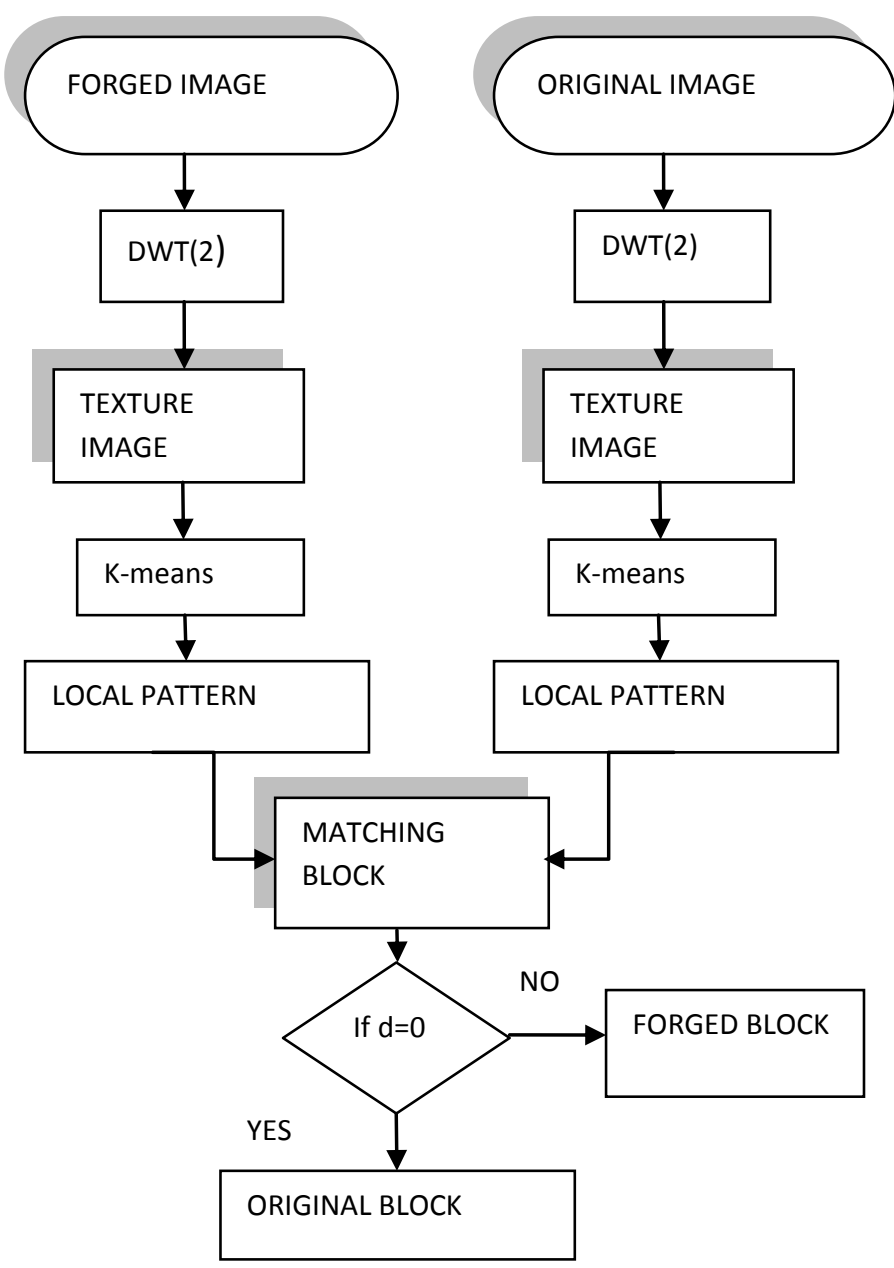

Figure 2 proposed model for image forged image

Step 1 . Initially put the original image and forged image for the processing of feature extraction

Step 2. After processing of image discrete wavelet transform function are applied for the texture feature extraction

Step 3. After the texture feature extraction apply k-means technique for local pattern generation

Step 4. The pattern matching block selects the all local pattern of cluster algorithm of both original and forged image

Step 5. Measure the distance between original image and forged image.

Step 6 If the value of $d$ is 0 images are block is original else image block area is forged

\section{EXPERIMENTAL RESULT ANALYSIS}

To evaluate the performance of proposed method of image forgery detection we have use MATLAB software 7.8.0 with a variety of image dataset used for experimental task [17,18]. Firstly we get the values of Detection Rate and FRR through Feature Technique LDBM and then Proposed Technique after run the program code. Finally we get the better result of Proposed Technique with compare to Feature Technique. 
Performance evaluation and result analysis

Table1: Shows that the Detection Rate and FRR with using LDBM and Proposed method for the same and different number of images.

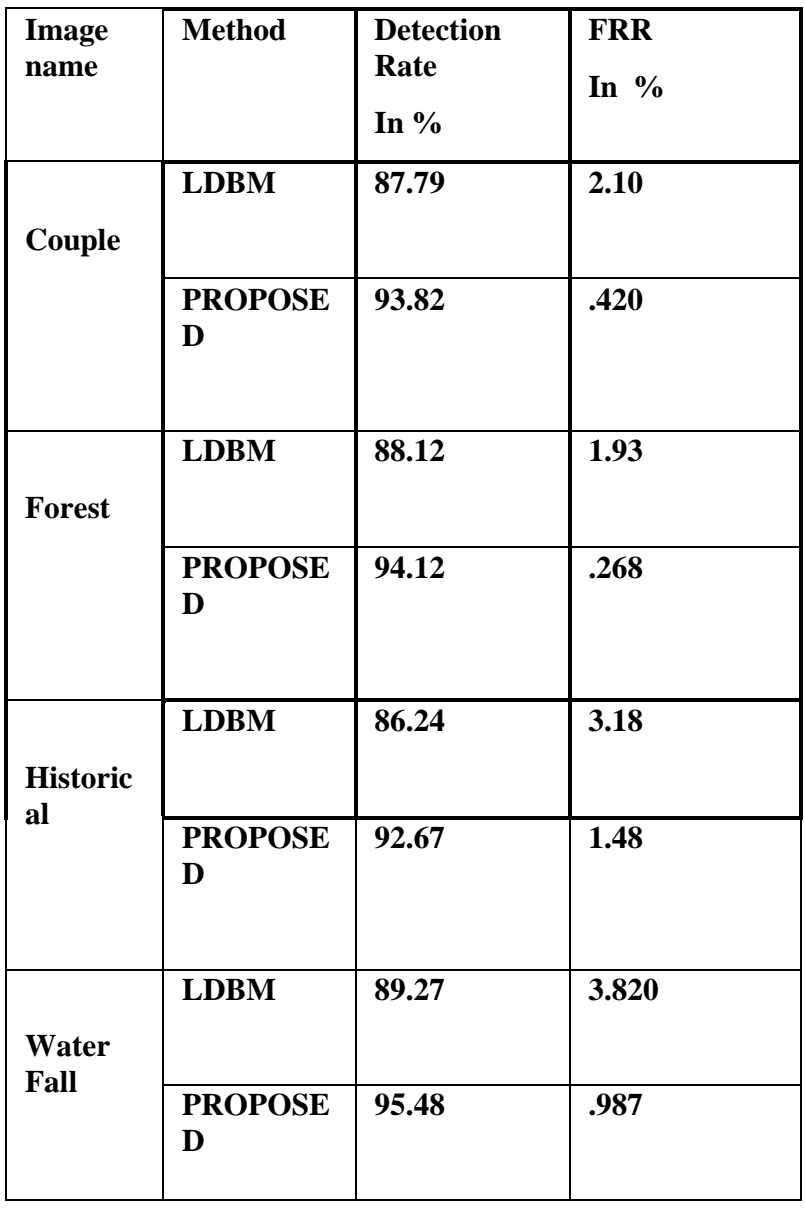

Comparative result graph for image foregery detection using LDBM and Proposed method for Coupled Image

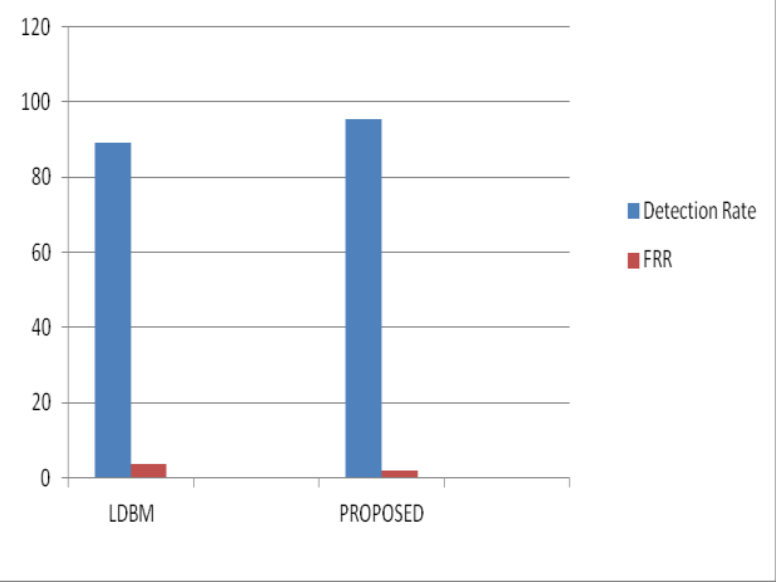

Figure 3: Shows that comparative result of Image "Coupled", with using LDBM and Proposed method and here our proposed method shows that the better result in the form of higher Detection Rate and low FRR than the existing method.
Comparative result graph for image foregery detection using LDBM and Proposed method for Forest Image

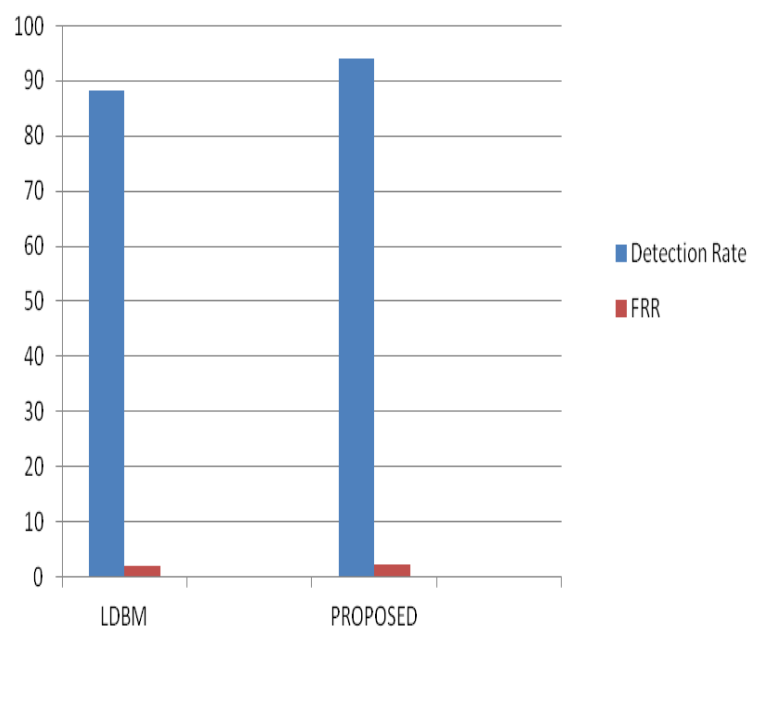

Figure 4: Shows that comparative result of Image "Forest", with using LDBM and Proposed method and here our proposed method shows that the better result in the form of higher Detection Rate and low FRR than the existing method.

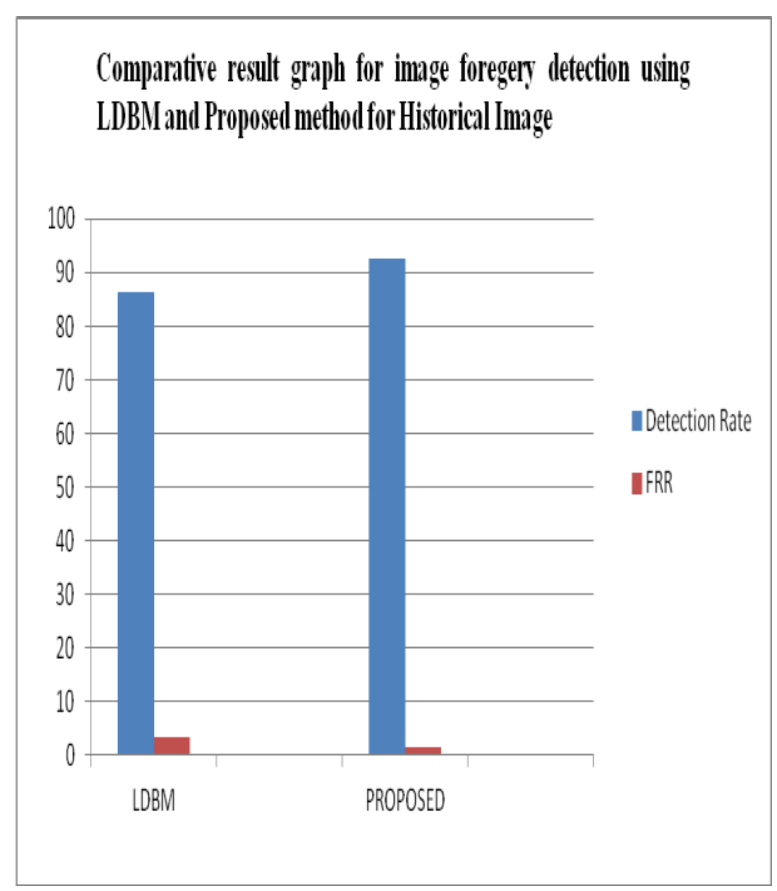

Figure 5: Shows that comparative result of Image "Historical", with using LDBM and Proposed method and here our proposed method shows that the better result in the form of higher Detection Rate and low FRR than the existing method. 


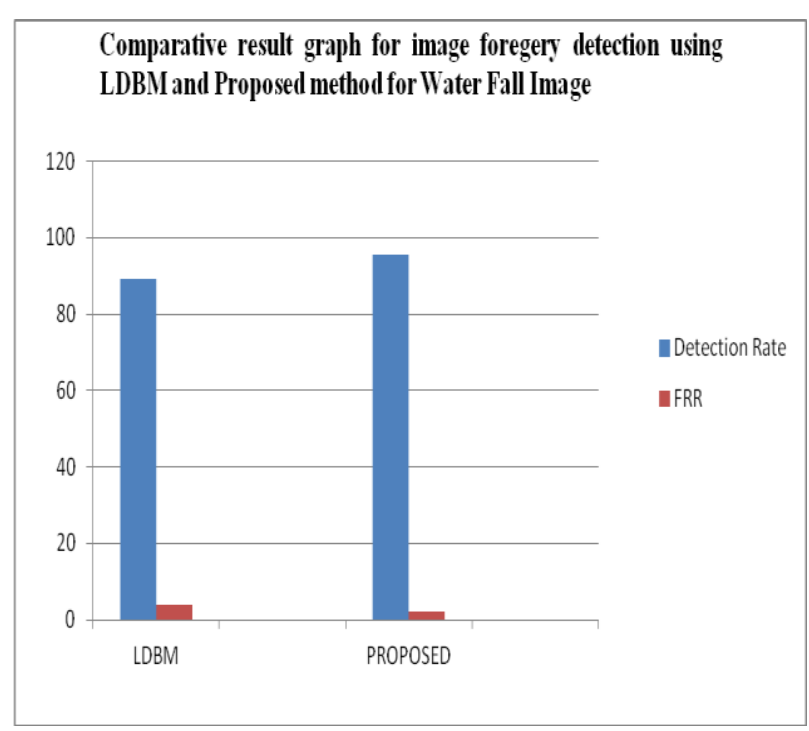

Figure 6: Shows that comparative result of Image "Water Fall" with using DWT and Proposed method and here our proposed method shows that the better result in the form of higher PSNR and low FRR than the existing method.

\section{CONCLUSION AND FUTURE WORK}

In this dissertation proposed an image forgery detection technique based on clustering technique. The proposed image forgery used wavelet transform function for the extraction of feature of original and forged image. The extracted feature passes through clustering technique for the generation of local pattern. The local pattern passes though matching block and measure distance of two similar and dissimilar blocks. The proposed image forged detection technique is very efficient in compression of local pattern and transform function based technique. The proposed methods are evaluated on a number of original and forged images. According to our experimental results the proposed methods are quite attractive. The forgery is done with just copy-move, copy-move with rotation, with scaling, and reflection. In this process, an image database that consists of original and forged images is also developed. The proposed method achieves 100\% accuracy in just copy-move forgery (without any change in the size or characteristics of the object) forgery without post-processing and $97.43 \%$, $66.58 \%$, and $99.12 \%$ accuracies in copy-move forgery with rotation, scaling, and reflection, respectively. Also to ensure more efficiency, we have added some random noise on the images, the detection accuracy achieved $98.23 \%$. While the proposed method performs well even with additive white Gaussian noise post-processing.

\section{REFERENCES}

[1] Abhishek Kashyap, Shiv Dutt Joshi, "Detection of CopyMove Forgery Using Wavelet Decomposition" IEEE, 2013, Pp 396-400.

[2] Saba Mushtaq and Ajaz Hussain Mir, "Digital Image Forgeries and Passive Image Authentication Techniques: A Survey" International Journal of Advanced Science and Technology (IJAST), 2014, Vol.73, Pp 15-32.

[3] Ketan S Bacchuwar, Aakashdeep, K.R Ramakrishnan, "A Jump Patch-Block Match Algorithm for Multiple Forgery Detection” IEEE, 2013, Pp 723-726.

[4] Ghulam Muhammada, Muhammad Hussain , George Bebis , "Passive Copy Move Image Forgery Detection using Undecimated Dyadic Wavelet Transform” Digital Investigation, 2012,Vol. 9,Pp 49-57.

[5] Sondos M. Fadl , Noura A. Semary, Mohiy M. Hadhoud, "Copy-Rotate-Move Forgery Detection Based on Spatial Domain" IEEE,2014,Pp 136-141.

[6] Cheng-Shian Lin and Jyh-Jong Tsay, " Passive Forgery Detection for JPEG Compressed Image based on Block Size Estimation and Consistency Analysis" Natural Science Publishing Cor.,2015, Pp 1015-1028.

[7] Michael Zimba,Sun Xingming, "DWT-PCA (EVD) Based Copy-move Image Forgery Detection" IJDCTA, Vol.5, 2011, Pp 251-258 .

[8] Ghulam Muhammad , Munner H, Al-Hammadi , Muhammad Hussain, George Bebis, "Image Forgery Detection using Steerable Pyramid Transform and Local Binary Pattern" Springer-Verlag Berlin Heidelberg ,2013.

[9] Giovanni Chierchia, Giovanni Poggi, Carlo Sansone, "A Bayesian-MRF Approach for PRNU-based Image Forgery Detection” IEEE, 2013. Pp 1-14.

[10] Tiziano Bianchi, Alessia De Rosa, Alessandro Piva, "Improved DCT Coefficient Analysis For Forgery Localization In JPEG Images" IEEE, 2011. Pp 24442447.

[11] Weiqi Luo, Zhenhua Qu, Jiwu Huang, Guoping Qiu, “A Novel Method For Detecting Cropped And Recompressed Image Block” IEEE, 2007, Pp 217-220.

[12] F. Battisti, M. Carli, A. Neri , "Image Forgery Detection by using No-Reference quality metrics"

[13] M. Barni, A. Costanzo and L. Sabatini, "Identification of Cut and paste Tampering by means of Double-JPEG Detection and Image Segmentation" IEEE, 2010, Pp 1687-1690.

[14] Zhang, Zhen, Zhou Yu and BaiNa Su., "Detection of Composite Forged Image" IEEE Int. Conf. Computer Application and System Modeling (ICCASM), Taiyuan, Oct. 2010, Vol. 11, Pp 572-576.

[15] H. Farid, "Image Forgery Detection" Signal Processing Magazine, IEEE, March 2009,Vol. 26, No. 2, Pp 16-25.

[16] [G. Cao, Y. Zhao, R. Ni and X. Li, "Contrast Enhancement-Based Forensics in Digital Images" IEEE Transactions on Information Forensics and Security, 2014,Vol 9, No. 3, Pp 515-525.

[17] G. Chierchia, G. Poggi, C. Sansone and L. Verdoliva, "A Bayesian-MRF Approach for PRNU-Based Image Forgery Detection" Information Forensics and Security, IEEE Transactions, 2014, Vol. 9, No. 4, Pp 554-567.

[18] G. K. Birajdar and V. H. Mankar, "Digital Image Forgery Detection using Passive Techniques: A survey" Digital investigations, 2013, Pp. 226-245.

[19] P. Xunyu and L. Siwei, "Region Duplication Detection using Image Feature Matching", IEEE Trans on Information Forensics and Security, 2011, Vol. 5, No. 4, Pp 857-67.

[20] P. Kakar and N. Sudha, "Exposing Post Processed Copypaste Forgeries through Transform-invariant Features", IEEE Trans. on Information Forensics and Security, 2012, Vol. 7, No. 3,Pp 1018-28. 